\title{
Joint PAPR Reduction Scheme in OFDM-PON Systems
}

\author{
Chunlei Zhang*, Kai Zhao and Xunfa Yang \\ School of Electronic and Information Engineering, Lanzhou Jiaotong University, Lanzhou, China \\ ${ }^{*}$ Corresponding author
}

\begin{abstract}
To meet the demands of different high bandwidth service, optical orthogonal frequency-division multiple accessbased passive optical network (OFDM-PON) has been considered as one of the candidate for NG-PON2. In this paper, a joint peakto-average power ration (PAPR) reduction scheme in OFDMPON system is proposed. The simulation results show that the system PAPR is greatly improved comparing with other methods. The required OSNR of the improved system decreases under the same BER, and the system performance is greatly improved.
\end{abstract}

Keywords-peak-to-average power ration (PAPR); passive optical network (PON); orthogonal frequency division multiplexing (OFDM); daptive predistortion

\section{INTRODUCTION}

With the development of high-bandwidth services, such as IPTV and video-on-demand, next generation passive optical network (NG-PON) has been regarded as a promising technology in order to meet access bandwidth requirement. OFDM-PON has been researched as a promising candidate for NG-PON2, due to its benefits such as strong dispersion tolerance, high spectral efficiency, flexible resource allocation and potentially low cost [1-4]. Nowdays, how to build a highquality access network is one of the key problem for NG-PON.

The basic concept of OFDM is that data are transmitted on a number of different parallel subcarriers; hence, the total data rate is divided by the number of the carriers and high speed data transmission can be achieved. Once the phase of all the subcarriers is the same,there will be a high peak amplitude; and this can introduce a very large However, the basic OFDM signal is of high peak to average power ration (PAPR), as the individual subcarrier signals add up coherently to produce high peaks in the time domain. The high PAPR requires optical componets with a wide linear range to accommodate for the signal variations and deteriorate the transmission performance of the OFDM signal [5-11]. In addition, scientific analysis has shown that four-wave mixing (FWM) is closely related to an instantaneous peak power. Because of the narrow frequency interval among subcarriers, the phase condition of fiber FWM can be easily mateched.Hence, high PAPR is one of main challenges in OFDM-PON systems.

In this paper, a novel joint PAPR reduction scheme is proposed. The paper is organized as follows. In section II, the system architecture of WDM-OFDM-PON using LDPC code is given. The performance of the proposed system is simulated in section III. The results show that the proposed scheme can greatly improve the transmission performance. At last, we conclude the paper.

\section{JOINT PAPR REDUCTION SCHEME WITH CLIPPING AND PRE-DISTORTION}

\section{A. PAPR in OFDM-PON Systems}

An OFDM signal is generated using multiple subcarriers transmitted in parallel. Simultaneous transmission of multiple subcarriers is a challenging task owing to the fact that this operation drastically increases the system complexity. This issue can be addressed by employing the Inverse Discrete Fourier Transformation (IDFT), also represented as Inverse Fast Fourier Transformation (IFFT) as the name given to it for the fast calculation of the transformation. OFDM signal contains $N$ orthogonal subcarriers. When $N$ subcarriers superimposed in the same direction, a large peak will produce. The maximum peak power is $N$ times the average power, and produce large PAPR. PAPR is defined as the ratio of the maximum instantaneous power of a symbol period over average power. It can be expressed as Equation 1.

$$
\operatorname{PAPR}(d B)=10 \lg \frac{\max \left(\left|x_{n}\right|^{2}\right)}{\mathrm{E}\left(\left|x_{n}\right|^{2}\right)}
$$

where $x_{\mathrm{n}}$ denotes the OFDM signals after inverse fast fourier transform (IFFT) defined as Equation 2 and E( ) denotes the operation of mathematical expectation.

$$
x_{n}=\frac{1}{\sqrt{N}} \sum_{k=0}^{N-1} X_{k} W_{N}^{n k}
$$

typically, the peak statistical characteristic of the OFDM signal can be analyzed by using complementary cumulative distribution function (CCDF) of the system PAPR. The CCDF can be expressed as Equation 3.

$$
P\{P A P R>P\}=1-P\{P A P R \leq P\}=1-\left(1-e^{-p}\right)^{N}
$$

Even PAPR may occur low, but in the OFDM-PON transmission system, the high PAPR has affected the transmission quality of the system signal and system performance. OFDM transmitter and the receiver of the HPA and $\mathrm{ADC}$ demand the greater linear range. The nonlinear of these non-linear devices will cause nonlinear distortion of inband signal and signal distortion [10], and also cause nonlinear effects of the fiber. In order to transmit high peak OFDM signal without distortion, we must adopt appropriate techniques to suppress PAPR of OFDM-PON system. 
Numerous methods have been proposed to reduce PAPR, such as peak windowing, clipping and filtering, selected mapping (SLM), partial transmit sequence (PTS), Reed-Muller codes, tone injection, interleaving, etc. the computation complexity, BER and data rate are some trade-offs of these techniques $[2,9,11]$. The existing methods of suppressing PAPR can be divided into three class: clipping, coding and probability.

\section{B. Principle of the Joint Scheme}

To reduce the PAPR in OFDM-PON system directly using conventional clipping method can cause in-band distortion and out-band radiation, leading the system BER performance degradation. Improved clipping methods can be divided into two parts: K-times oversampling, clipping and filtering. The basic principle is shown in Figure 1. First by K-times oversampling the aliasing, which is caused by all clipping noise coming in band, can effectively avoid; then clipping and filtering the OFDM signal, i.e. by FFT converting the signal from time domain to frequency domain. To suppress out-band radiation by setting zero in-band signals. Finally the signal is converted to the time domain by IFFT, clipping and filtering, completely filtering the out-band interference. But modified clipping method will lead the PAPR of OFDM signal to rise. Therefore, repeated clipping and filtering can be used to further suppress peak amplitude of the signal until satisfactory level.

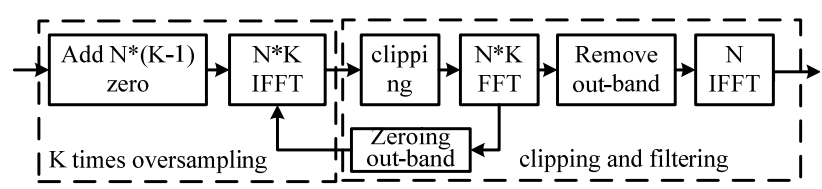

FIGURE I. PRINCIPLE DIAGRAM OF THE IMPROVED CLIPPING SCHEME

Predistortion technique is a simple and effective linear method to eliminate non-linear, that is, before the non-linear device a nonlinear unit is added to compensate for the distortion caused by the nonlinear device [8]. In this paper, adaptive predistortion method is employed to eliminate nonlinear of the OFDM-PON system. However linear range of predistortion is limited, when the linear output value corresponding to the input voltage of the power amplifier is greater than the maximum output voltage of the power amplifier, predistortion cannot compensate nonlinear distortion of the system. To ensure the linear of the system, assuming bias of the MZM is $V_{\pi / 2}, V_{\pi}$ is half-wave voltage, the input of the pre-distortion $X_{i n}$ and the output of the predistortion signal $V_{\text {out }}$ should be met with Equation 4 .

$$
V_{\text {out }}=\frac{V_{\pi}}{\pi} \cos ^{-1}\left(\sqrt{\frac{X_{\text {in }}}{\max \left\{\left|X_{\text {in }}\right|\right\}}}\right)
$$

Our scheme joint the modified clipping and adaptive predistortion to suppress PAPR of the system. The system schematic diagram of the transmitting side is shown in Figure II PAPR of the signals is suppressed by the modified clipping method. When the signal amplitude is at the non-linear range of the power amplifier, the distortion cannot be adjusted with pre-distortion, direct clipping and filtering is employed. The signal with greater peak power is pre-distorted to make the amplitude of amplifier's input signal does not exceed the saturation voltage value, which means the power amplifier

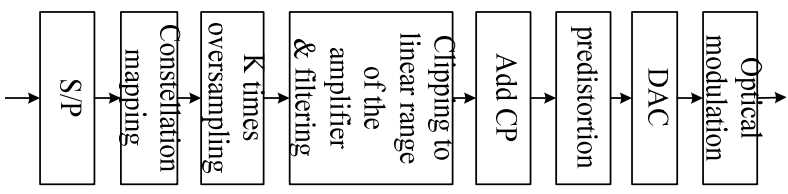

FIGURE II. PRINCIPLE DIAGRAM OF THE OFDM-PON TRANSMITTER

within the dynamic range. Before entering the power amplifier, the signal is linearized by predistortion processing to increase the linear range of the amplifier. This can effectively reduce out-band radiation of the signal, and ultimately reduce the inband loss. When the amplitude of the input signal is small, it is input to the amplifier directly. The clipping ratio is constantly adjusted to achieve the best working conditions. The system segment range of the joint approach is shown in Figure III.

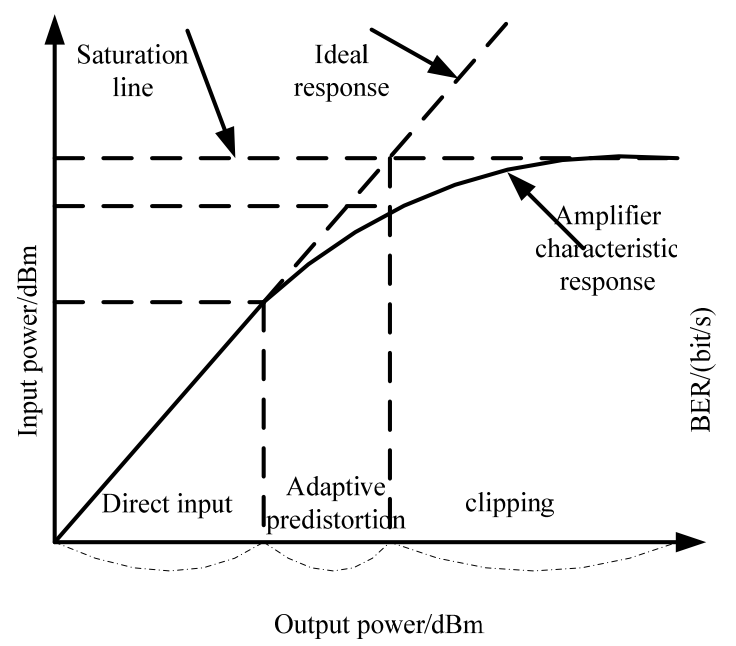

FIGURE III. SYSTEM SEGMENT RANGE OF THE JOINT SCHEME

\section{System Architecture}

The optical OFDM system includes coherent optical OFDM (CO-OFDM) and direct detection optical OFDM (DDO-OFDM). We use direct detection system to receive in our system considering the transmission distance and the system costs. The OFDM-PON system employing the proposed joint modified clipping is illustrated in Figure 4. The system consists OLT and ONUs. The difference of our proposed system is at the transmitter compared with common system, which process the signal of OFDM transmitter with the proposed scheme to reduce the PAPR of the OFDM signal.

In the OLT, the data are sent to the downlink OFDM transmitter module. In this module data are modulated into OFDM data, the modulated data change to downlink RF OFDM signal by the up-converter, and finally the signal goes through the optical intensity modulator to achieve electrical to optical conversion, generating downstream optical OFDM 
signal. The downstream optical signal passes through an optical circular (OC) to send to the fiber channel downlink optical link transmitted to ONUs. Optical signal is divided into a plurality of beams through a splitter, and finally sent to each ONU.

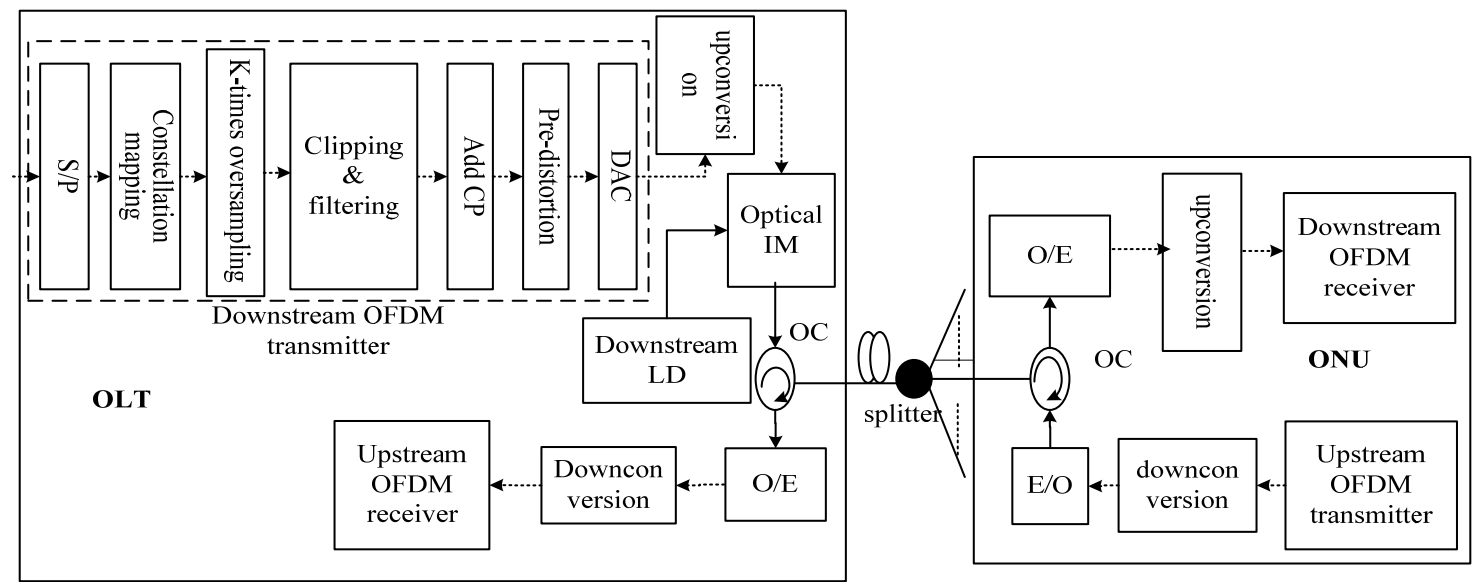

FIGURE IV. OFDM-PON SYSTEM WITH JOINT IMPROVED SCHEME

\section{SimULATION RESULTS}

In this section, we verified the OFDM-PON downstream transmission performance of the system with proposed scheme. The OFDM signal suppressed PAPR is generated in MatLab, the optical transmission performance is simulated by Optisystem.

OFDM signals map using 4 QAM. The number of subcarriers is set 128 , and the cyclic prefix (CP) is 16 . The oversampling factor is 4 . The simulation results show as in Figure 5. Figure 5 illustrates the PAPR curves of original OFDM signal and the joint improved scheme with different filtering times. $\mathrm{M}$ represents the iterated filtering times. When $\mathrm{CCDF}=10^{-4}$, the PAPR of the original OFDM signal is $11.8 \mathrm{~dB}$; while when the $m=2$ using clipping and filtering process, the PAPR equals $7.7 \mathrm{~dB}$. When $m=4$, the PAPR reduced to $6.5 \mathrm{~dB}$. From the curve, we can see that joint improved method is more effective for PAPR suppression than the traditional and improved clipping, and the greater the number of iterations $m$ for improved clipping, the better the effect of suppressing the PAPR.

Also we analyzed the downstream transmission performance of OFDM-PON system shown as Figure 6 and Figure 7, respectively to without and with the joint improved PAPR reduction scheme. The center frequency of the CW laser is $193.1 \mathrm{THz}$, and the length of the single mode fiber is $50 \mathrm{~km}$ in the system. The BER curves of the downlink without our proposed scheme are compared in Figure 6. For the OFDM-PON system without joint improved scheme when BER is $10^{-4}$, the back-to-back OSNR equals $13.9 \mathrm{~dB}$; and when the transmitting power is $-8 \mathrm{dBm}$, the OSNR is $16.1 \mathrm{~dB}$; when transmitting power is $-4 \mathrm{dBm}$, the OSNR is $16.5 \mathrm{~dB}$; as the transmitting power is $0 \mathrm{dBm}$, the OSNR is $22 \mathrm{~dB}$. It can be seen that the greater the transmitting power, the greater the PAPR and causing OSNR of the system required increased, the poorer system performance.
Simulation results for the OFDM-PON system with joint improved scheme are shown in Figure 7. Also we compare downlink BER performance for back-to-back and with different transmitting powers of $0 \mathrm{dBm},-4 \mathrm{dBm}$ and $-8 \mathrm{dBm}$ respectively. When BER is $10^{-4}$, the back-to-back required OSNR equals $15.1 \mathrm{~dB}$; and when the transmitting power equals $-8 \mathrm{dBm}$, the OSNR is $15.1 \mathrm{~dB}$; and when the transmitting power is $-4 \mathrm{dBm}$, the OSNR is $15.5 \mathrm{~dB}$; as the transmitting power is OdBm, the OSNR is $17.8 \mathrm{~dB}$. Contrast the above data can be seen, when the transmitting power is $0 \mathrm{dBm}$, the required OSNR of system with joint improved scheme reduces $4.2 \mathrm{~dB}$ than the system without using joint improvement scheme. In addition, in the system without joint improved scheme when BER is $10^{-4}$, required OSNR of the system with $0 \mathrm{dBm}$ transmitting power is $5.9 \mathrm{~dB}$ greater than $-8 \mathrm{dBm}$ transmitting power; while in the OFDM-PON system with the joint improved scheme, the required OSNR is only $2.7 \mathrm{~dB}$ greater. There is $3.2 \mathrm{~dB}$ improvement.

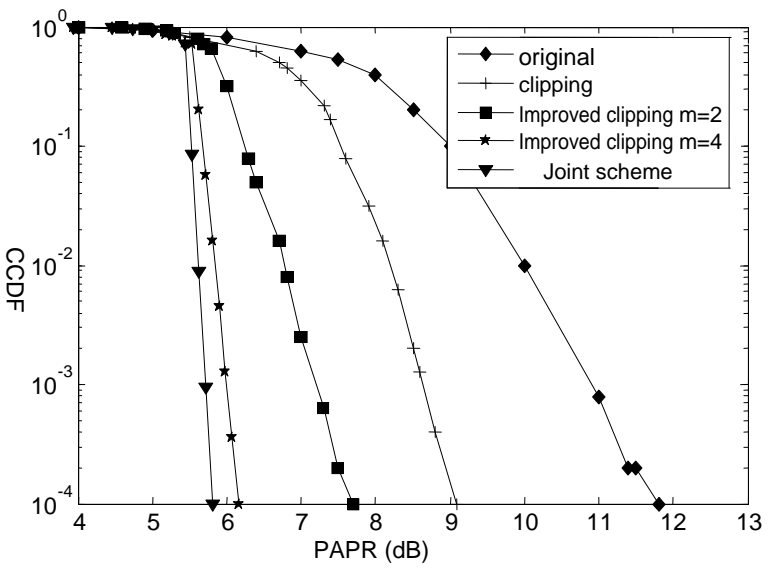

FIGURE V. PAPR PERFORMANCE OF OFDM SYSTEM PON 


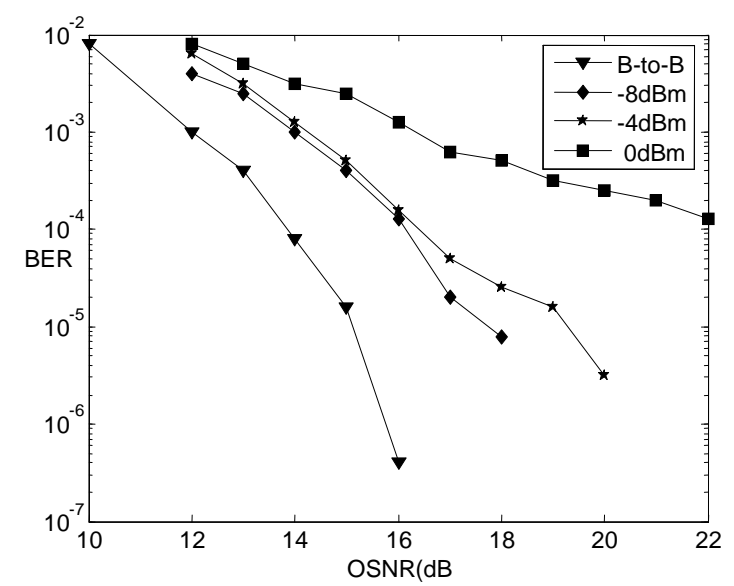

FIGURE VI. SYSTEM BER PERFORMANCE OF OFDM-PON

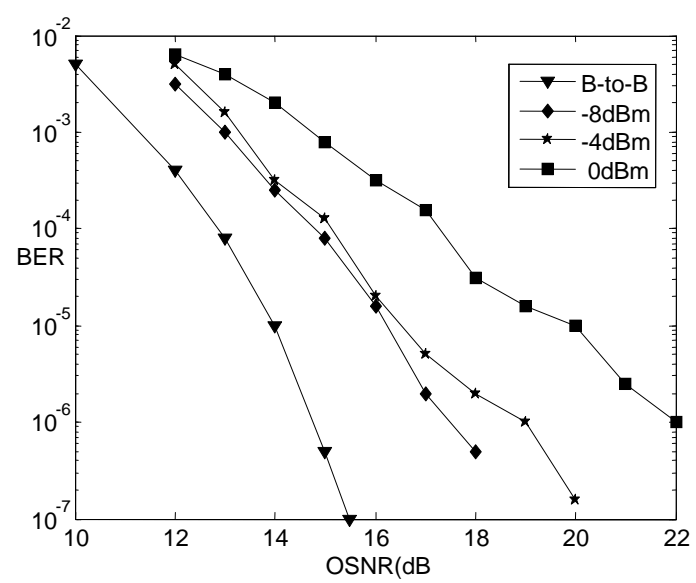

FIGURE VII. SYSTEM BER PERFORMANCE WITH DIFFERENT CODING SCHEMES

From the above analysis we can see, the joint improved method can suppress the nonlinear of the system, thereby improving system performance. And as the transmission power increases, the performance of downstream transmission system with joint improved method improves more obvious.

\section{CONCLUSION}

PAPR reduction in OFDM system is a challenge and various techniques have been suggested previously. In this paper, we have proposed the joint improved clipping and filtering PAPR reduction scheme in OFDM-PON. The obtained simulation results prove that the scheme can reduce PAPR of the optical OFDM signal and suppress the nonlinear effect of the system. The required OSNR of the system decreases comparing with system without improved clipping and predistoration, and the system performance improves greatly especially at $0 \mathrm{dBm}$.

\section{ACKNOWLEDGMENT}

This work is partly supported by Natural Science Funds of Gansu Province under Contract No.148RJZA047. First Author is corresponding author.

\section{REFERENCES}

[1] D. Nesset, "NG-PON2 technology and standards," Journal of Lightwave Technology, vol. 33, no. 5, pp. 1136-1143, Mar. 2015.

[2] W. O. Popoola, Z. Ghassemlooy, and B. G. Stewart, "Pilot-assisted PAPR reduction technique for optical OFDM communication systems," Journal Lightwave Technology, vol. 32, no. 7, pp. 1374-1382, Apr. 2014.

[3] Cheng Ju, Xue Chen, Na Liu, Qi Zhang, and Huitao Wang, “OFDM PON downstream scheme with symbol pre-distortion and scalable receiver frontend,” IEEE Optical Fiber Communications Conference and Exhibition (OFC) 2015, Th1H.4.

[4] Wei Zhang, Chongfu Zhang, Chen Chen, Wei Jin, and Kun Qiu, “Joint PAPR reduction and physical layer security enhancement in OFDMAPON,” IEEE Photonics Technology Letters, vol. 28, no. 9, pp. 998-1001, May 2016.

[5] A. Tsokanos, E. Giacoumidis, G. Zardas, A. Kavatzikidis, N.P. Diamantopoulos, I. Aldaya, I. Tomkos, "Reductions of peak-to-average power ratio and optical beat interference in cost-effective OFDMAPONs,” Photonic Network Communications, vol. 26, no.2, pp.44-52, 2013.

[6] Maria C. Yuang, Po-Lung Tien, Dar-Zu Hsu, Shing-Yu Chen, ChiaChien Wei, Ju-Lin Shih, and Jyehong Chen, "A High-Performance OFDMA-PON System Architecture and Medium Access Control," Journal of Lightwave Technology, vol. 30, no. 11, pp.1685-1693, 2012.

[7] Yanir London and Dan Sadot, "Nonlinear Effects Mitigation in Coherent Optical OFDM System in Presence of High Peak Power,” Journal of Lightwave Technology, vol. 29, no. 21, pp. 3275-3281, 2011.

[8] Jiang Yang,Yuan Min, Chen Biyun, and Wang Quan, "Modified united algorithm for readucing PAPR of MIMO-OFDM system,” Application Research of Computers, vol. 29, no. 1, pp. 250-252, 2012.

[9] SHIEH W, DJORDJEVIE I, OFDM for Optical Communications, Burlington, Elsevier, 2010.

[10] Yu Wang, Tao Fang, Lap-Pui Chau, and Kim-Hui Yap, "Twodimensional channel ending scheme for MCTF-based scalable video coding,” IEEE Transactions On Multimedia, vol. 9, no. 1, pp. 37-45, 2007.

[11] Nan Jingchang, Qu Yun, and Mao Luhong, "ation of PAPR reduction and pre-distortion in OFDM-RoF system," Optical Communication Technology, vol. 36, no. 10, pp. 57-59, 2012. 